\title{
Greatest of the Least Primes in Arithmetic Progressions Having a Given Modulus
}

\author{
By Samuel S. Wagstaff, Jr.
}

\begin{abstract}
We give a heuristic argument, supported by numerical evidence, which suggests that the maximum, taken over the reduced residue classes modulo $k$, of the least prime in the class, is usually about $\phi(k) \log k \log \phi(k)$, where $\phi$ is Euler's phi-function.
\end{abstract}

1. Introduction. When $(k, l)=1$, let $P(k, l)$ denote the least prime in the arithmetic progression $l+k n, n \geqslant 0$. Let $P(k)=\max _{l} P(k, l)$, where the maximum is taken over all $l$ for which $0<l<k$ and $(k, l)=1$.

In 1944, Linnik [5] proved that $P(k, l)<k^{A}$ for some large absolute constant $A$. Since then, several authors have found successively smaller, but still large, explicit values for $A$. Titchmarsh showed (Theorem 6 of [11]) that the Extended Riemann Hypothesis (ERH) for $L$-functions of characters modulo $k$ implies that $P(k, l) \ll$ $(\phi(k))^{2}(\log k)^{4}$. Kanold [3] conjectured that $P(k, l)<k^{2}$ always. Heath-Brown [2] remarked that presumably $P(k, l) \ll k(\log k)^{2}$. Turán [12], also assuming the ERH, proved that for each $\delta>0$, we have

$$
P(k, l)<\phi(k) \log ^{2+\delta} k
$$

for almost all residue classes $l$, i.e., the inequality fails for at most $o(\phi(k))$ of the $l$ 's as $k \rightarrow \infty$. His result, in contrast to the preceding statements, says nothing about $P(k)$. We mention it here because we will argue below that (1) holds for all $l$.

In the other direction, Landau (see Section 62 of [4]) proved the elementary result $P(k)>k+1$ for every $k>30$. From the Prime Number Theorem it is clear that for every positive $\epsilon$, we have $P(k)>(1-\epsilon) \phi(k) \log k$ for all $k>K(\epsilon)$. Erdös [1] proved that there is an $\epsilon>0$ so that $P(k)>(1+\epsilon) \phi(k) \log k$ for infinitely many $k$ 's. Prachar [8] and Schinzel [10] have shown that there is a $c>0$ so that for every $l$ there are infinitely many $k$ for which $P(k, l)>c k \log k \log _{2} k \log _{4} k /\left(\log _{3} k\right)^{2}$, where $\log _{r} k$ is the $r$-fold iterated logarithm. Pomerance [7] has shown that

$$
P(k)>\left(e^{\gamma}-o(1)\right) \phi(k) \log k \log _{2} k \log _{4} k /\left(\log _{3} k\right)^{2}
$$

except for a set of $k$ 's of asymptotic density zero.

In summary, it is known that $P(k)$ almost always exceeds $\phi(k) \log k$ and is always less than $k^{A}$ for some large $A$, but even the ERH does not seem to yield $P(k)=o\left(k^{2}\right)$. We will present a heuristic argument that $P(k)$ is usually close to $\phi(k) \log k \log \phi(k)$ for large $k$. Since $\log \phi(k) \sim \log k$ as $k \rightarrow \infty$, our reasoning says also that $P(k)$ is

Received August 25, 1978; revised September 22, 1978.

AMS (MOS) subject classifications (1970). Primary $10 \mathrm{H} 20$.

Key words and phrases. Least prime in an arithmetic progression.

(C) 1979 American Mathematical Society 0025-5718/79/0000-0116/\$03.00 
approximately $\phi(k) \log ^{2} k$ for most large $k$. We retain the $\log \phi(k)$ in the expression because it arises naturally in the argument and because the formula with it fits the numerical data slightly more closely.

2. The Heuristic Estimate of $P(k)$. Let $X=m k \log k$, where $m=m(k)$ will be chosen later. The number of positive integers below $X$ and relatively prime to $k$ is about $X \phi(k) / k$, and about $X / \log X$ of these are prime. Thus, the conditional probability that a number below $X$ is prime, given that it is relatively prime to $k$, is approximately $k /(\phi(k) \log X)$. If $0<l<k$ and $(k, l)=1$, then the probability that all $[X / k]$ of the numbers

$$
l, l+k, l+2 k, \ldots, l+[m \log k-1] k
$$

(omitting $l$ if $l=1$ ) are composite is about

$$
\left(1-\frac{k}{\phi(k) \log X}\right)^{m \log k} \text {. }
$$

If we now require that $m<\log ^{3} k$, say, so that $\log X \sim \log k$ as $k \rightarrow \infty$, then the latter probability is approximately

$$
\left(1-\frac{k}{\phi(k) \log k}\right)^{m \log k} \approx e^{-m k / \phi(k)} .
$$

A. For any $l$, note that $X$ will be a rough approximation to $P(k, l)$ if we choose $m$ just large enough so that the probability (2) is neither very close to 0 nor to 1 , say, $e^{-m k / \phi(k)} \approx e^{-c}$, where $c$ is some positive constant of moderate size. We find $m \approx$ $c \phi(k) / k$, so that $P(k, l) \approx X \approx c \phi(k) \log k$. This estimate is consistent with the results of Erdös [1] and may be obtained more directly by noting that there are about $c$ primes below $c \phi(k) \log k$ in each of the classes relatively prime to $k$.

B. To estimate $P(k)$ by $X$, we want $m$ to be so large that each residue class prime to $k$ will have a good chance of containing a prime below $X$. From (2), the probability that every class does contain one (assuming independence of the classes) is about

$$
\left(1-e^{-m k / \phi(k)}\right)^{\phi(k)} .
$$

As in $\mathrm{A}$, we ask that this probability be $\approx e^{-c}$. Then

$$
e^{-m k / \phi(k)} \approx 1-e^{-c / \phi(k)} \approx c / \phi(k)
$$

or

$$
P(k) \approx X \approx \phi(k) \log k(\log \phi(k)-\log c) \sim \phi(k) \log k \log \phi(k)
$$

as $k \rightarrow \infty$. This time $c$ becomes less and less significant as $k$ increases. This is the desired estimate of $P(k)$.

To obtain greater assurance that every class contains at least one prime, we could let $c \rightarrow 0$ as $k \rightarrow \infty$. With $c=1 / \log k$, for example, we see that $P(k)<$ $(1+\epsilon) \phi(k) \log k \log \phi(k)$ for almost all large $k$. A similar argument with $c=\log k$ shows that $P(k)>(1-\epsilon) \phi(k) \log k \log \phi(k)$ for almost all large $k$. This reasoning suggests, indeed, that if we disregard a small set (i.e., of asymptotic density zero) of exceptions, then $P(k) \sim \phi(k) \log k \log \phi(k)$ as $k \rightarrow \infty$. 
TABLE 1

Typical values of $P(k)$

\begin{tabular}{|c|c|c|c|c|c|}
\hline $\mathrm{k}$ & $\phi(k)$ & $\ell$ & $P(k)$ & $\mathrm{P}(\mathrm{k}) /$ est & {$[P(k) / k$} \\
\hline 11 & 10 & 10 & 43 & 0.78 & 3 \\
\hline 12 & 4 & 1 & 13 & 0.94 & 1 \\
\hline 13 & 12 & 12 & 103 & 1.35 & 7 \\
\hline 14 & 6 & 1 & 29 & 1.02 & 2 \\
\hline 15 & 8 & 1 & 31 & 0.69 & 2 \\
\hline 1000 & 400 & 921 & 13921 & 0.84 & 13 \\
\hline 1001 & 720 & 74 & 33107 & 1.01 & 33 \\
\hline 1002 & 332 & 391 & 13417 & 1.01 & 13 \\
\hline 1003 & 928 & 822 & 43951 & 1.00 & 43 \\
\hline 1004 & 500 & 371 & 18443 & 0.86 & 18 \\
\hline 10000 & 4000 & 7461 & 477461 & 1.56 & 47 \\
\hline 10001 & 9792 & 3382 & 873469 & 1.05 & 87 \\
\hline 10002 & 3332 & 9937 & 209977 & 0.84 & 20 \\
\hline 10003 & 8568 & 5919 & 806159 & 1.13 & 80 \\
\hline 10004 & 4800 & 4565 & 374713 & 1.00 & 37 \\
\hline 49996 & 24080 & 37163 & 2236987 & 0.85 & 44 \\
\hline 49997 & 46784 & 32740 & 5582407 & 1.03 & 111 \\
\hline 49998 & 15360 & 1813 & 1301761 & 0.81 & 26 \\
\hline 49999 & 49998 & 13525 & 6213401 & 1.06 & 124 \\
\hline 50000 & 20000 & 15219 & 2615219 & 1.22 & 52 \\
\hline 97651 & 97650 & 92811 & 12787441 & 0.99 & 130 \\
\hline 97652 & 48824 & 62001 & 6897641 & 1.14 & 70 \\
\hline 97653 & 63504 & 24581 & 9985187 & 1.24 & 102 \\
\hline 97654 & 48360 & 76247 & 5935487 & 0.99 & 60 \\
\hline 97655 & 78120 & 56096 & 9723941 & 0.96 & 99 \\
\hline 510521 & 464100 & 388050 & 80539847 & 1.01 & 157 \\
\hline 510522 & 170172 & 384341 & 29994617 & 1.11 & 58 \\
\hline 510523 & 466464 & 504724 & 83719973 & 1.05 & 163 \\
\hline 510524 & 218784 & 123199 & 33307259 & 0.94 & 65 \\
\hline 510525 & 272160 & 420388 & 37688713 & 0.84 & 73 \\
\hline 999996 & 330672 & 764329 & 54764113 & 0.94 & 54 \\
\hline 999997 & 997920 & 532429 & 222531703 & 1.17 & 222 \\
\hline 999998 & 480060 & 382501 & 89382323 & 1.03 & 89 \\
\hline 999999 & 466560 & 550894 & 71550823 & 0.85 & 71 \\
\hline .000000 & 400000 & 434781 & 72484781 & 1.02 & 72 \\
\hline
\end{tabular}

3. The Numerical Evidence. Perhaps the foregoing heuristic argument can be improved. In any case, we can report that the empirical results given below do show that $P(k) / \phi(k) \log k \log \phi(k)$ is usually near 1 . We give special attention to the exceptional $k$ below, listing all those where this ratio is not so close to 1 . We computed $P(k)$ for many values of $k$. Let $S$ be the set of integers $k$ in the intervals $11 \leqslant k \leqslant$ $50000,95001 \leqslant k \leqslant 100000,510501 \leqslant k \leqslant 510550$, and $999951 \leqslant k \leqslant 1000000$, together with $k=115147,357819$, and 636184 . For each $k$ in $S$, we computed $P(k)$ and recorded the $l$ for which $P(k, l)$ is greatest. We could not extend the calculation beyond 1000000 because of space and time limitations. The block of 50 numbers beginning with 510501 was included because $510510=2 \cdot 3 \cdot 5 \cdot 7 \cdot 11 \cdot 13 \cdot 17$ is the 
TABLE 2

Examples with small ratio $P(k) /$ est

$\begin{array}{rrrrcc}k & \phi(k) & \ell & P(k) & P(k) / e s t & {[P(k) / k]} \\ 102 & 32 & 95 & 197 & 0.384 & 1 \\ 150 & 40 & 143 & 293 & 0.396 & 1 \\ 210 & 48 & 209 & 419 & 0.422 & 1 \\ 130 & 48 & 129 & 389 & 0.430 & 2 \\ 51 & 32 & 44 & 197 & 0.452 & 3 \\ & & & & & \\ 420 & 96 & 361 & 1201 & 0.454 & 2 \\ 228 & 72 & 77 & 761 & 0.455 & 3 \\ 240 & 80 & 145 & 883 & 0.458 & 3 \\ 312 & 96 & 217 & 1153 & 0.458 & 3 \\ 75 & 40 & 68 & 293 & 0.460 & \\ & & & & & 3 \\ 110 & 40 & 1 & 331 & 0.477 & 3 \\ 105 & 48 & 104 & 419 & 0.485 & 4 \\ 462 & 120 & 323 & 1709 & 0.485 & 3 \\ 528 & 160 & 361 & 2473 & 0.486 & \\ 570 & 144 & 511 & 2221 & 0.489 & \end{array}$

TABLE 3

Examples with large ratio $P(k) /$ est

$\begin{array}{rrrrlcl}\mathrm{k} & \phi(\mathrm{k}) & \ell & \mathrm{P}(\mathrm{k}) & \mathrm{P}(\mathrm{k}) / \text { est } & {[\mathrm{P}(\mathrm{k}) / \mathrm{k}]} & \text { Factorization } \\ 1623 & 1080 & 1478 & 123203 & 2.209 & 75 & 3 \cdot 541 \\ 636154 & 315840 & 629991 & 116415479 & 2.178 & 182 & 8 \cdot 281 \cdot 283 \\ 461 & 460 & 22 & 37363 & 2.160 & 81 & 461 \\ 23636 & 11160 & 9451 & 2183963 & 2.085 & 92 & 4 \cdot 19 \cdot 311 \\ 3246 & 1080 & 3101 & 123203 & 2.020 & 37 & 2 \cdot 3 \cdot 541 \\ & & & & & & \\ 1945 & 1552 & 722 & 169937 & 1.968 & 87 & 5 \cdot 389 \\ 10948 & 4224 & 7989 & 642973 & 1.960 & 58 & 4 \cdot 7 \cdot 17 \cdot 23 \\ 922 & 460 & 483 & 37363 & 1.941 & 40 & 2 \cdot 461 \\ 4095 i & 39600 & 38984 & 8352037 & 1.876 & 203 & 31 \cdot 1321 \\ 19515 & 10560 & 3058 & 1806223 & 1.866 & 91 & 3 \cdot 5 \cdot 1321 \\ 32844 & 8448 & 27521 & 1472657 & 1.854 & 44 & 4 \cdot 3 \cdot 7 \cdot 17 \cdot 23 \\ 22505 & 15408 & 7419 & 2753029 & 1.849 & 122 & 5 \cdot 7 \cdot 643 \\ 541 & 540 & 18 & 39511 & 1.848 & 73 & 541 \\ 2732 & 1364 & 1587 & 143651 & 1.844 & 52 & 4 \cdot 683 \\ 7049 & 5616 & 4420 & 786859 & 1.832 & 111 & 7 \cdot 19 \cdot 53\end{array}$

number below 1000000 for which $\phi(k) / k$ is least. We computed $P(k)$ for the three special values of $k$ because we thought there was a reason (see below) why $P(k)$ might be unusually large for these $k$. The nine cases $2 \leqslant k \leqslant 10$ are left as an exercise for the reader.

Table 1 illustrates typical results from the huge table in our possession. The particular intervals of length 5 given here were chosen to be representative of the nearby numbers. In each table, "est" stands for the estimate $\phi(k) \log k \log \phi(k)$. It appears that, in fact, the larger $k$ is, the more likely $P(k) /$ est is to be close to 1 . The column headed $l$ gives the (necessarily unique) $l$ for which $P(k, l)=P(k)$. The last column $[P(k) / k]$ gives the number of composites before the first prime in the arithmetic progression $l+k n$. 


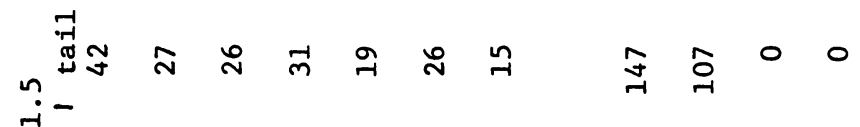

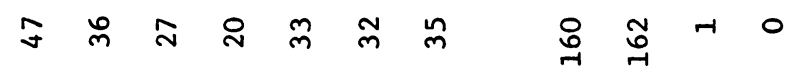

$$
\begin{aligned}
& \stackrel{+}{-i}- \\
& \infty \text { ล ผ } \\
& \text { i- } \\
& \stackrel{-1}{-1}
\end{aligned}
$$

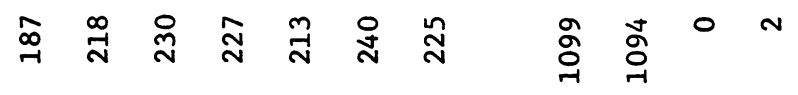

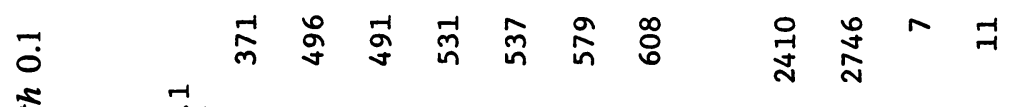

$$
\begin{aligned}
& \text { 胥 }
\end{aligned}
$$

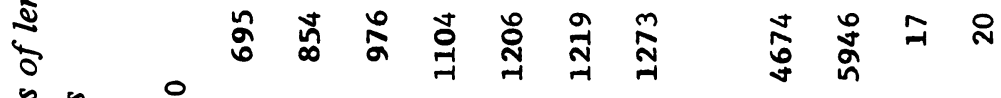

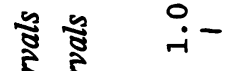

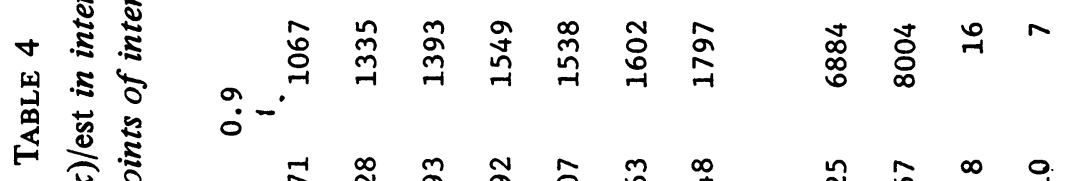

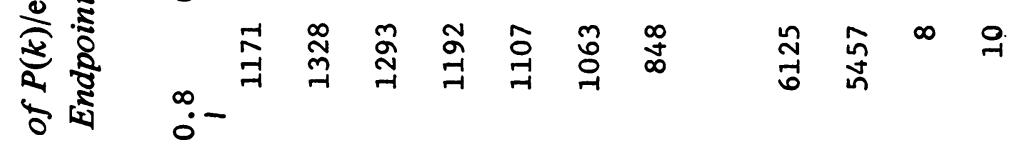

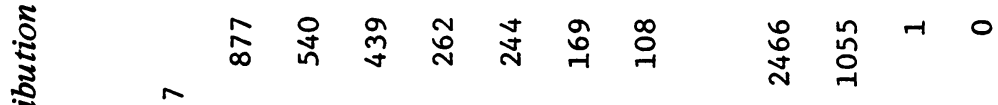

$$
\begin{aligned}
& \text { í- } \\
& \text { in } N \text { i }
\end{aligned}
$$

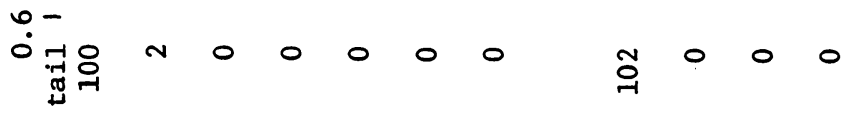

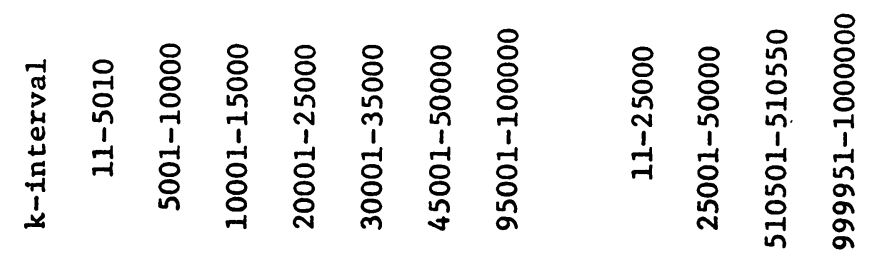


In Tables 2 and 3 we list the 15 moduli $k \in S$ for which $P(k)$ /est is smallest and greatest. Note that the $k$ 's in Table 2 are all small, while several quite large numbers $k$ appear in Table 3. This happens because a single bad arithmetic progression can inflate $P(k)$, but a very great deal of good luck is required for every one of the $\phi(k)$ progressions to contain a small prime. Note also that $102=2 \cdot 51,150=2 \cdot 75,210=$ $2 \cdot 105,3246=2 \cdot 1623$, and $922=2 \cdot 461$, and that in each case the same progression causes that large $P(k)$ for both the odd number and its double. Moreover, $1623=$ $3 \cdot 541$, but different progressions inflate $P(k)$ for 1623 and 541 .

The heuristic argument predicts that the distribution of $P(k) / \phi(k) \log k \log \phi(k)$ should have mean 1 and should peak more and more sharply about 1 as $k$ increases. Table 4, which gives the distribution of this ratio for seven intervals of 5000 values of $k$ each, supports this prediction well. (The statistics for four other intervals, two longer and two shorter than 5000, are also given at the end of Table 4.) The two center intervals of length 0.1 contain more and more of the ratios as the size of $k$ increases. The size of the tails continually decreases. We have already explained why the upper tail is larger than the lower tail. The mean values of the ratio seem to converge slowly to 1 .

Low [6] and Purdy [9] found three values of $k$ for which a certain Dirichlet $L$-function $L_{-k}(s)$ with modulus $k$ comes very close to 0 at $s=1 / 2$. Although there is no known connection between $P(k)$ and $L_{-k}(1 / 2)$, we once thought that a small value of the latter would cause a large value of the former. It was at that time that we computed $P(k)$ for $k=115147,357819$, and 636184 . The first two $k$ produced ordinary ratios $P(k) / \phi(k) \log k \log \phi(k)$ of 1.20 and 1.03 , but the third modulus did have the unusually high ratio shown in Table 3 . The modulus 636184 deserves more study to determine if there really is a connection.

We became interested in studying $P(k, l)$ during the calculations of [13], where we had to compute $P(p, 1)$ for many primes $p$. We observed there that $P(p, 1)$ is about $p \log p$ on the average, which agrees with $\mathrm{A}$ of Section 2. The values of $P(p)$ for $p \in S$ seem to be statistically indistinguishable from those for $P(k)$ for all $k \in S$. There is nothing special about a prime modulus (except, of course, that $\phi(p)=p-1$ ). For very small $k$, the value 1 often appears as the $l$ for which $P(k)=P(k, l)$. For $k>500$, however, the residue class $1(\bmod k)$ is almost never the one with the greatest least prime. In fact, the numbers $l / k$, where $P(k, l)=P(k)$, seem to have a uniform distribution in the unit interval $(0,1)$.

After he conjectured that $P(k) / \phi(k) \log k$ tends to infinity as $k \rightarrow \infty$, Pomerance [7] remarked that, in proving a lower bound for $P(k)$, the hardest values of $k$ to treat seem to be the products of the first $r$ primes for various $r$. We see from Table 5 that $P(k) / \phi(k) \log k \log \phi(k)$ really is a bit low for these numbers. Since the $k$ 's in Table 2 have only small prime divisors, it would be tempting to conjecture that the ratio will always be small for such numbers. However, the ratio is 1.352 for $k=675=3^{3} \cdot 5^{2}$ and 1.960 for $k=10948=2^{2} \cdot 7 \cdot 17 \cdot 23$. The numbers whose prime divisors are as small as possible are the powers of 2 . Some of these are listed in Table 5. The ratios for these $k$ are a little low, but definitely not unusually so. 


\section{TABLE 5}

\begin{tabular}{rrrrrr}
\multicolumn{7}{c}{$P(k)$ for $k=2 \cdot 3 \cdot 5 \cdots \cdots \cdot p_{r}$ and $k=2^{n}$} \\
$\mathrm{k}$ & $\phi(\mathrm{k})$ & $\ell$ & $\mathrm{P}(\mathrm{k})$ & $\mathrm{P}(\mathrm{k}) /$ est & {$[\mathrm{P}(\mathrm{k}) / \mathrm{k}]$} \\
30 & 8 & 1 & 31 & 0.548 & 1 \\
210 & 48 & 209 & 419 & 0.422 & 1 \\
2310 & 480 & 697 & 14557 & 0.634 & 6 \\
30030 & 5760 & 14111 & 464561 & 0.903 & 13 \\
510510 & 92160 & 126449 & 10336649 & 0.747 & 20 \\
& & & & & \\
16 & 8 & 9 & 41 & 0.889 & 3 \\
32 & 16 & 1 & 97 & 0.631 & 4 \\
64 & 32 & 57 & 313 & 0.679 & 6 \\
128 & 64 & 85 & 853 & 0.660 & 14 \\
256 & 128 & 125 & 3709 & 1.077 & 15 \\
512 & 256 & 1 & 7681 & 0.867 & 18 \\
1024 & 512 & 441 & 20921 & 0.945 & 25 \\
2048 & 1024 & 1939 & 38803 & 0.717 & 39 \\
4096 & 2048 & 4017 & 106417 & 0.819 & 38 \\
8192 & 4096 & 2159 & 321647 & 1.048 &
\end{tabular}

The maximum of $P(k) / k$ with $k \in S$ occurred at $k=510533$. The ratio was 247.98 , $l=499932$, and $P(k)=126601583$. Among the first 50000 numbers $k$, the greatest $P(k) / k$ was 204.98 at $k=47903$, with $P(k)=9819037$ and $l=46825$.

The author warmly thanks Professor Douglas Hensley for a valuable discussion of the heuristic argument. He is grateful to the Computing Services Office of the University of Illinois for letting him use so much computer time.

Department of Mathematics

University of Illinois at Urbana-Champaign

Urbana, Illinois 61801

1. P. ERDÖS, "On some applications of Brun's method," Acta Sci. Math. (Szeged), v. 13, 1949, pp. 57-63.

2. D. R. HEATH-BROWN, "Almost-primes in arithmetic progressions and short intervals," Math. Proc. Cambridge Philos. Soc., v. 83, 1978, pp. 357-375.

3. H.-J. KANOLD, “Über Primzahlen in arithmetischen Folgen," Math. Ann., v. 156, 1964, pp. 393-395.

4. E. LANDAU, Handbuch der Lehre von der Verteilung der Primzahlen, Band I, Teubner, Leipzig-Berlin, 1909. Reprinted by Chelsea, New York, 1953.

5. U. V. LINNIK, "On the least prime in an arithmetic progression. I. The basic theorem," Rec. Math. (N.S.), v. 15 (57), 1944, pp. 139-178.

6. M. E. LOW, "Real zeros of the Dedekind zeta function of an imaginary quadratic field," Acta Arith., v. 14, 1968, pp. 117-140.

7. C. POMERANCE, "A note on the least prime in an arithmetic progression." (To appear.)

8. K. PRACHAR, "Über die kleinste Primzahl einer arithmetischen Reihe," J. Reine Angew. Math., v. 206, 1961, pp. 3-4.

9. G. B. PURDY, Some Extremal Problems in Geometry and the Theory of Numbers, Ph.D. thesis, University of Illinois at Urbana-Champaign, 1972. 
10. A. SCHINZEL, "Remark on the paper of K. Prachar "Über die kleinste Primzahl einer arithmetischen Reihe',"J. Reine Angew. Math., v. 210, 1962, pp. 121-122.

11. E. C. TITCHMARSH, “A divisor problem," Rend. Circ. Mat. Palermo, v. 54, 1930, pp. 414-429.

12. P. TURÁN, "Über die Primzahlen der arithmetischen Progression," Acta Sci. Math. (Szeged), v. 8, 1936/37, pp. 226-235.

13. S. S. WAGSTAFF, JR., "The irregular primes to 125000," Math. Comp., v. 32, 1978, pp. 583-591. 